\title{
La croissance du bovin Azawak au Niger : influence de facteurs de variation non génétiques et estimation des paramètres génétiques
}

\author{
Seyni Siddo ${ }^{(1,2)}$, Nassim Moula ${ }^{(2,3)}$, Issa Hamadou ${ }^{(1,2)}$, Moumouni Issa ${ }^{(4)}$, \\ Hamani Marichatou ${ }^{(4)}$, Nicolas Antoine-Moussiaux ${ }^{(2,3)}$, Pascal Leroy ${ }^{(2,3)}$, \\ Charles Michaux ${ }^{(2,5)}$
}

\author{
(1) Institut National de la Recherche Agronomique du Niger. Département des Productions animales. BP 429. Niamey \\ (Niger). \\ (2) Université de Liège. Faculté de Médecine vétérinaire. Département des Productions animales. Boulevard de Colonster, 20. \\ Bâtiment B43. BE-4000 Liège (Belgique).E-mail : nassim.moula@uliege.be \\ (3) Université de Liège. Faculté de Médecine vétérinaire. Institut vétérinaire tropical. Boulevard de Colonster, 20. \\ Bâtiment B43. BE-4000 Liège (Belgique). \\ (4) Université Abdou Moumouni. Faculté d'Agronomie. Département des Productions animales. BP 10960. Niamey (Niger). \\ (5) Université libre de Bruxelles (ULB). Faculté de Médecine. Route de Lennik, 808. BE-1070 Bruxelles (Belgique).
}

Reçu le 18 septembre 2016, accepté le 16 avril 2018, mis en ligne le 8 mai 2018.

Cet article est distribué suivant les termes et les conditions de la licence CC-BY (http://creativecommons.org/licenses/by/4.0/ deed.fr)

Description du sujet. Les effets des facteurs non génétiques sur les caractères de croissance du bovin Azawak au Niger et les paramètres génétiques sont estimés à partir des performances enregistrées sur des animaux élevés dans la station de Toukounous.

Objectifs. Grâce aux résultats obtenus, un modèle de prédiction des valeurs d'élevage de candidats reproducteurs améliorateurs sera développé.

Méthode. Les facteurs non génétiques ont été évalués grâce à des modèles linéaires fixes et les paramètres génétiques grâce à un modèle animal multi-caractère par la méthode REML.

Résultats. Les effets du sexe, de la saison et de l'année de naissance et leurs interactions sont significatifs $(p<0,05)$ sur tous les poids étudiés, à la naissance et de 12 à 20 mois et sur les gains moyens quotidiens de la naissance à 12 (GMQ12) et 18 (GMQ18) mois. L'héritabilité est modérée pour le poids à la naissance $(0,20)$ et à 12,14 et 20 mois $(0,15$ à 0,43$)$. Elle est élevée pour le poids à 13 mois $(0,61)$, le GMQ12 $(0,58)$ et le GMQ18 $(0,44)$. Les corrélations génétiques entre les poids et les gains sont faibles à élevées et elles se réduisent progressivement avec l'espacement des pesées, mais elles sont positives à l'exception de celles entre le GMQ12 et le poids à 17 mois et le GMQ12 et GMQ18. Les corrélations environnementales suivent les mêmes tendances que les corrélations génétiques.

Conclusions. L'héritabilité des poids et des gains est modérée à élevée, une amélioration génétique pourrait être obtenue par la sélection sur les performances de croissance.

Mots-clés. Bovin Azawak, croissance, facteurs non génétiques, paramètres génétiques, Niger.

\section{Effects of genetic and non-genetic factors on growth performance in Azawak cattle in Niger}

Description of the subject. The effects of both non-genetic factors and of genetic parameters on the growth traits of Azawak cattle in Niger were estimated from performance data collected on animals raised in the Toukounous animal breeding station. Objectives. Following on from the results obtained, a model to predict breeding values of breeding stocks is to be developed. Method. The influence of non-genetic factors and of genetic parameters was estimated using a fixed effects linear model and a multi character animal model (REML), respectively.

Results. Sex, season and year of birth, and their interactions had a significant effect $(p<0.05)$ on weight from birth to 20 months and average daily gains from birth to 12 months (ADG12) and to 18 months (ADG18). Heritability was moderate for birth weight (0.20) and for weight at 12,14 and 20 months (0.15 to 0.43), whereas it was higher for weight at 13 months $(0.61)$, 
ADG12 (0.58) and ADG18 (0.44). Genetic correlations between weights and gains were low to high, and they decreased gradually as the interval between weighing increased. All genetic correlations were positive except those between ADG12 and weight at 17 months and between ADG12 and ADG18. Environmental correlations followed the same trends as genetic correlations.

Conclusions. Heritability of weight and gains was moderate to high, indicating that an improvement in meat production could be obtained from selection according to growth performance.

Keywords. Azawak cattle, growth, non-genetic factors, genetic parameters, Niger.

\section{INTRODUCTION}

La croissance pondérale est un caractère important dans la gestion et/ou la sélection des espèces animales d'élevage. Elle est le reflet de leur capacité d'adaptation aux conditions environnementales et de leur valeur économique (Thiruvenkadan et al., 2009). L'évolution pondérale est le principal critère d'appréciation reflétant l'adaptation à un environnement difficile où l'alimentation, l'abreuvement et la pression sanitaire sont des facteurs limitant de la productivité (Njoya et al., 1998). De plus, le poids est un indicateur important du revenu des producteurs des animaux à viande (Gunawan \& Jakaria, 2011).

Au Niger, le zébu Azawak, adapté aux conditions environnementales sahéliennes, bénéficie d'un intérêt grandissant de la part de l'État nigérien et d'un grand nombre d'éleveurs. La mise en œuvre d'un programme d'amélioration génétique et la diffusion du progrès génétique nécessitent d'abord une évaluation de l'effet des facteurs non génétiques enregistrés sur les caractères d'intérêt, afin d'éliminer leur biais lors de l'estimation des paramètres génétiques de ces mêmes caractères. Mais les études sur le zébu Azawak sont rares, notamment dans son berceau qui est le Niger. Même s'il fait l'objet d'une sélection depuis 1954 à la station de Toukounous, dont les principaux objectifs sont la robe de couleur fauve, les productions de lait et de viande et qu'il bénéficie de plus en plus d'une amélioration génétique dans la sous-région où il est reconnu pour ses aptitudes mixtes (viande et lait) (Ouedraogo et al., 2008), on ne dispose guère, concernant la croissance pondérale, que de paramètres génétiques estimés en 1982 (Chartier et al., 1982; Achard \& Chanono, 1995). Le but de la présente étude est d'évaluer, chez des bovins Azawak élevés à la station de Toukounous, l'effet des facteurs non génétiques sur les caractères de croissance comprenant le poids à la naissance et les poids entre 12 et 20 mois, et les gains moyens quotidiens (GMQ) entre la naissance d'une part et les âges de 12 et 18 mois d'autre part, et d'estimer les paramètres génétiques de ces mêmes caractères grâce à un modèle incluant les facteurs environnementaux dont l'effet s'est avéré être significatif afin d'en éliminer le biais.

\section{MATÉRIEL ET MÉTHODES}

\subsection{Milieu de l'étude}

L'étude a été conduite à la station de Toukounous qui est implantée à environ $22 \mathrm{~km}$ de la commune urbaine de Filingué, chef-lieu du Département de Filingué, et à $200 \mathrm{~km}$ au nord-est de Niamey, la capitale du Niger, située entre $14^{\circ} 31^{\prime}$ Nord et $3^{\circ} 18^{\prime}$ Est. La station a une superficie de 45000 ha, dont 4400 ha de terres de parcours, avec une capacité de charge initiale de 1100 têtes (soit 1056 unités de bétail tropical, UBT). Actuellement, la capacité de charge est de 447 UBT. Les terres de parcours sont organisées en cinq parcs, qui sont subdivisés en 30 parcelles de surfaces variables clôturées par des barbelés. La station est établie sur des sols sableux à limono-argileux.

Le climat est de type sahélien. Il est caractérisé par deux saisons principales. La saison des pluies s'étend de juin à septembre, sa durée est variable d'une année à l'autre avec une durée maximale de 105 jours, la pluviosité moyenne est de $350 \mathrm{~mm} \cdot \mathrm{an}^{-1}$. La saison sèche dure généralement neuf mois avec deux composantes : une saison sèche froide d'octobre à février où les températures ne dépassent guère $20^{\circ} \mathrm{C}$ et une saison sèche chaude de mars à juin où la température moyenne peut atteindre $36{ }^{\circ} \mathrm{C}$ avec des pics à $45^{\circ} \mathrm{C}$. Durant cette dernière période, les conditions alimentaires sont particulièrement précaires. La végétation annuelle est constituée de graminées (Schoenefeldia gracilis, Aristida mitabilis, Cenchrus bijlorus) et d'une strate arbustive composée principalement de trois espèces Maerua crassifolia, Boscia senegalensis, Boscia angustifolia (Douma et al., 2007).

\subsection{Les animaux et le mode d'élevage}

L'étude porte sur les zébus Azawak de la station de Toukounous qui constituent le noyau améliorateur à partir duquel le progrès génétique est diffusé dans les troupeaux villageois. L'effectif du cheptel de la station s'élève à 700 têtes de bovins. Pour ce qui est de la production de viande, la sélection s'opère sur les caractères de croissance, poids et gain sur la base d'un testage sur la descendance.

Dès la naissance, chaque veau reçoit un matricule (année et numéro d'ordre de naissance dans l'année). 
Les veaux sont laissés sous la mère durant la première semaine pour consommer le colostrum ; par la suite, ils sont allaités uniquement avant les traites durant $1 \mathrm{~h}$ à $1 \mathrm{~h} 30$ deux fois par jour. Après le sevrage à 10 mois, l'allotement se fait selon le sexe, l'âge, le stade physiologique et l'option de production de l'animal (lait, viande ou mixité). Les informations nécessaires sont obtenues lors du contrôle hebdomadaire des performances zootechniques (âge et poids au sevrage, âge à la reproduction) et des caractères physiologiques (mise bas, gestation, chaleurs).

La mise en reproduction est effectuée à deux ans (pour les mâles et les femelles) et l'âge au premier vêlage est en moyenne de 36,5 mois (Achard \& Chanono, 1995).

La reproduction conduite en monte naturelle, à raison d'un taureau pour 50 à 60 vaches reproductrices, est répartie en quatre périodes : deux en saison sèche et froide (120 jours), de février à mars et de novembre à décembre, et deux en saison de pluie (90 jours), de juin à mi-juillet et de mi-aout à septembre. Ce programme de reproduction permet de répartir les naissances sur toute l'année afin de maintenir la production laitière de la station continue. Les inséminations artificielles (IA) se pratiquent à titre expérimental tout au long de l'année depuis 2007.

Le mode d'élevage est extensif. Les animaux s'alimentent sur des parcours naturels subdivisés en parcelles fourragères clôturées qui ne bénéficient d'aucune fumure. Le chargement des pâturages avoisine 1 UBT (qui équivaut à un bovin de $250 \mathrm{~kg}$ ) pour 6 ha. Pendant la saison sèche, les conditions alimentaires sont particulièrement précaires et les arbustes, toujours verts, complètent par leurs apports en matières azotées, vitamines et oligoéléments, une ration de paille de très faible valeur nutritionnelle. La production fourragère est très variable selon les années et les types de sols (Achard \& Chanono, 1995). En cas de baisse de la production fourragère, seules les vaches en lactation reçoivent des suppléments à base de grains ou de tourteau de coton de l'ordre de 0,4 à $2,2 \mathrm{~kg}$ par vache par jour selon le niveau de la production laitière.

La lutte préventive contre les épizooties consiste en des déparasitages interne et externe (les traitements contre les parasites externes sont conduits chaque semaine) et en vaccinations contre certaines maladies infectieuses (péripneumonie contagieuse bovine, pasteurellose, charbon) selon le calendrier officiel de prophylaxie du Niger. Les traitements curatifs sont administrés en cas de signes cliniques avérés.

\subsection{La collecte des données}

Les données collectées à la station de Toukounous sur les animaux nés au cours des années 1993 à 2012 sont : le numéro de l'animal, la date de naissance, le numéro du père, le numéro de la mère, le sexe, le rang de vêlage de la mère, le poids à la naissance et les poids mensuels depuis l'âge de 12 mois jusqu'à 25 mois. Néanmoins, ces performances et les effets du milieu n'ont pas été collectés de manière systématique. Les pesées après 12 mois n'ont pu être enregistrées qu'à partir de 2002. Ainsi, on dispose de 3870 poids à la naissance et de seulement 696 poids à 20 mois. Étant donné les faibles effectifs au-delà de 20 mois, les analyses ont porté sur les pesées mensuelles des poids de la naissance à 20 mois (P0 à P20) et sur les gains moyens quotidiens de zéro à 12 mois (GMQ12) et de zéro à 18 mois (GMQ18). Le gain entre 12 et 18 mois, que l'on pourrait considérer comme l'expression de la croissance post sevrage, n'a pas été analysé, car il n'a pu être calculé que chez 380 animaux, par défaut de l'un des deux poids. En outre, son coefficient de variation était de $99,5 \%$.

\subsection{Analyse des données}

Évaluation de l'effet des facteurs non génétiques sur les caractères de croissance. Les effets du sexe, de la saison et de l'année de naissance et de leurs interactions ont été étudiés à l'aide du logiciel SAS (procédure GLM ; SAS, 2008). Le modèle linéaire à effets fixes suivant a été utilisé pour l'analyse :

$\mathrm{Y}_{\mathrm{ijk} l}=\mu+\mathrm{a}_{\mathrm{j}}+\mathrm{b}_{\mathrm{k}}+\mathrm{c}_{1}+(\mathrm{ab})_{\mathrm{jk}}+(\mathrm{bc})_{\mathrm{kl}}+(\mathrm{ac})_{\mathrm{jl}}+(\mathrm{abc})_{\mathrm{jkl}}+\varepsilon_{\mathrm{ijk} l}$

où $\mathrm{Y}_{\mathrm{ij} \text { jkl }}$ est le poids ou GQM de l'animal $\mathrm{i}$, de sexe $\mathrm{j}$, né en saison $\mathrm{k}$ de l'année $1 ; \mu$ est la moyenne générale ; $a_{j}$ est l'effet fixe du sexe $(j:$ mâle ou femelle $) ; b_{k}$ est $l^{\prime}$ 'effet fixe de la saison de naissance ( $\mathrm{k}$ : saison froide, saison chaude et saison des pluies) ; $c_{1}$ est l'effet fixe de l'année de naissance (1: 1993 à 2012); $\mathrm{ab}_{\mathrm{jk}}$ est l'effet fixe de l'interaction sexe et saison de naissance ; $\mathrm{bc}_{\mathrm{k} 1}$ est l'effet fixe de l'interaction année et saison de naissance ; $\mathrm{ac}_{\mathrm{jl}}$ est l'effet fixe de l'interaction sexe et année de naissance ; $\mathrm{abc}_{\mathrm{jkl}}$ est l'effet fixe de l'interaction sexe - année de naissance - saison de naissance ; $\varepsilon_{\mathrm{ijkl}}$ est l'erreur résiduelle aléatoire.

Estimation des paramètres génétiques. Un modèle animal multi-caractère a été ajusté aux données et les composants de la variance et de la covariance des caractères étudiés ont été estimés par la méthode REML grâce au logiciel VCE 6.0.2 (Kovac et al., 2008). Dans ce modèle, l'interaction sexe - saison de naissance année de naissance, qui prend en compte l'ensemble des facteurs de variation identifiés précédemment, est considérée comme le seul effet fixe, le veau étant l'effet aléatoire. L'expression mathématique du modèle en notation matricielle était la suivante :

$$
\mathrm{Y}=\mathrm{Xb}+\mathrm{Za}+\varepsilon
$$


où $\mathrm{Y}$ est la matrice des poids à la naissance, des poids mensuels de 12 à 20 mois et des GMQ entre la naissance et 12 mois et la naissance et 18 mois ; X, Z sont les matrices qui associaient respectivement les vecteurs $b$ et a avec Y, la matrice $\mathrm{Z}$ comportait 5185 animaux ; a est le vecteur des effets génétiques aléatoires du veau ; b est le vecteur de l'interaction sexe du veau - saison de naissance - année de naissance et $\varepsilon$ est l'erreur résiduelle aléatoire.

\section{RÉSULTATS}

\subsection{Effets des facteurs non génétiques}

La statistique descriptive des caractères de croissance est présentée dans le tableau 1. Les analyses ont porté sur 3870 poids à la naissance, sur 1929 poids à 12 mois et sur un nombre variable de poids de 13 à 20 mois, l'effectif le plus élevé étant de 971 poids à 16 mois.

Les résultats de l'analyse de variance sont donnés au tableau 2, de même que les seuils de signification statistique des différences.

L'effet du sexe est significatif ( $p<0,001)$, les mâles ont des poids significativement supérieurs à ceux des femelles à tous les âges, de la naissance jusqu'à 20 mois, il en est de même pour les GMQ12 et GMQ18.

La saison de naissance a un effet significatif sur les poids de la naissance à 19 mois $(p<0,05)$, sauf à 13 et à 20 mois. Les GMQ sont aussi influencés significativement par la saison. Les veaux nés en saison des pluies ont des poids significativement $(p<0,05)$ plus élevés que ceux nés en saisons froide et chaude. Par contre, les veaux nés en saison froide présentent un
GMQ12 plus élevé $(p<0,05)$ que ceux nés en saisons pluvieuse et chaude. Le GMQ18 des veaux nés en saison chaude est plus élevé $(p<0,05)$ que celui des veaux nés en saisons froide et pluvieuse.

L'année de naissance influence significativement $(p<0,05)$ tous les poids et les GMQ. Le poids à la naissance baisse de 1994 à 1998 et se stabilise autour de $22 \mathrm{~kg}$ (Figure 1a). Le poids à 12 mois chute de 1993 à 2003, remonte ensuite et se stabilise autour de $160 \mathrm{~kg}$ (Figure 1b). Les autres poids suivent la même tendance que le poids à 12 mois entre 2002 et 2012 (Figure 1c). Les GMQ12 et GMQ18 sont les plus élevés respectivement en 2007 (406 g) et 2010 (375 g), alors que les minima sont notés en 2003 avec des valeurs respectives de $284 \mathrm{~g}$ et $247 \mathrm{~g}$.

L'interaction entre le sexe et la saison de naissance est significative $(p<0,01)$ pour les poids à $15,16,18$, et 19 mois et pour le GMQ18 (Tableau 2). Les veaux mâles nés en saison froide sont significativement $(p<0,001)$ plus lourds à 12 mois que ceux nés en saison sèche chaude et en saison des pluies. À 18 mois, ce sont les femelles nées en saison des pluies qui pèsent plus $(p<0,001)$ que celles nées en saison sèche chaude et froide. Les veaux mâles nés en saison sèche chaude présentent respectivement des GMQ12 et GMQ18 significativement $(p<0,05)$ plus importants. Les veaux mâles nés en saison froide et chaude présentent respectivement des GMQ12 et GMQ18 significativement $(p<0,05)$ plus importants. Ces gains ont été principalement réalisés au cours des saisons sèche et froide et des pluies, généralement favorables aux pâturages.

L'interaction entre l'année et la saison est significative $(p<0,01)$ pour tous les caractères de

Tableau 1. Statistique descriptive des caractères de croissance-Descriptive statistics of the growth traits.

\begin{tabular}{lccccc}
\hline Caractère & Nombre & Minimum & Maximum & Moyenne & Écart-type \\
\hline P0 $(\mathrm{kg})$ & 3870 & 10 & 34 & 22,3 & 1,9 \\
P12 $(\mathrm{kg})$ & 1929 & 76 & 279 & 156,2 & 30,0 \\
P13 $(\mathrm{kg})$ & 711 & 80 & 259 & 153,6 & 30,8 \\
P14 $(\mathrm{kg})$ & 809 & 79 & 270 & 163,9 & 33,6 \\
P15 $(\mathrm{kg})$ & 897 & 90 & 299 & 170,9 & 33,9 \\
P16 (kg) & 971 & 90 & 323 & 177,7 & 36,0 \\
P17 (kg) & 958 & 90 & 349 & 183,8 & 38,4 \\
P18 (kg) & 904 & 90 & 326 & 189,5 & 38,2 \\
P19 (kg) & 804 & 100 & 375 & 201,5 & 39,0 \\
P20 (kg) & 696 & 113 & 344 & 212,5 & 35,3 \\
GMQ12 $(\mathrm{g})$ & 1806 & 168 & 701 & 365,7 & 80,9 \\
GMQ18 $(\mathrm{g})$ & 849 & 120 & 554 & 305,5 & 69,5 \\
\hline P0
\end{tabular}

P0 à P20 : poids de la naissance à 20 mois - weight from the birth to 20 months. 


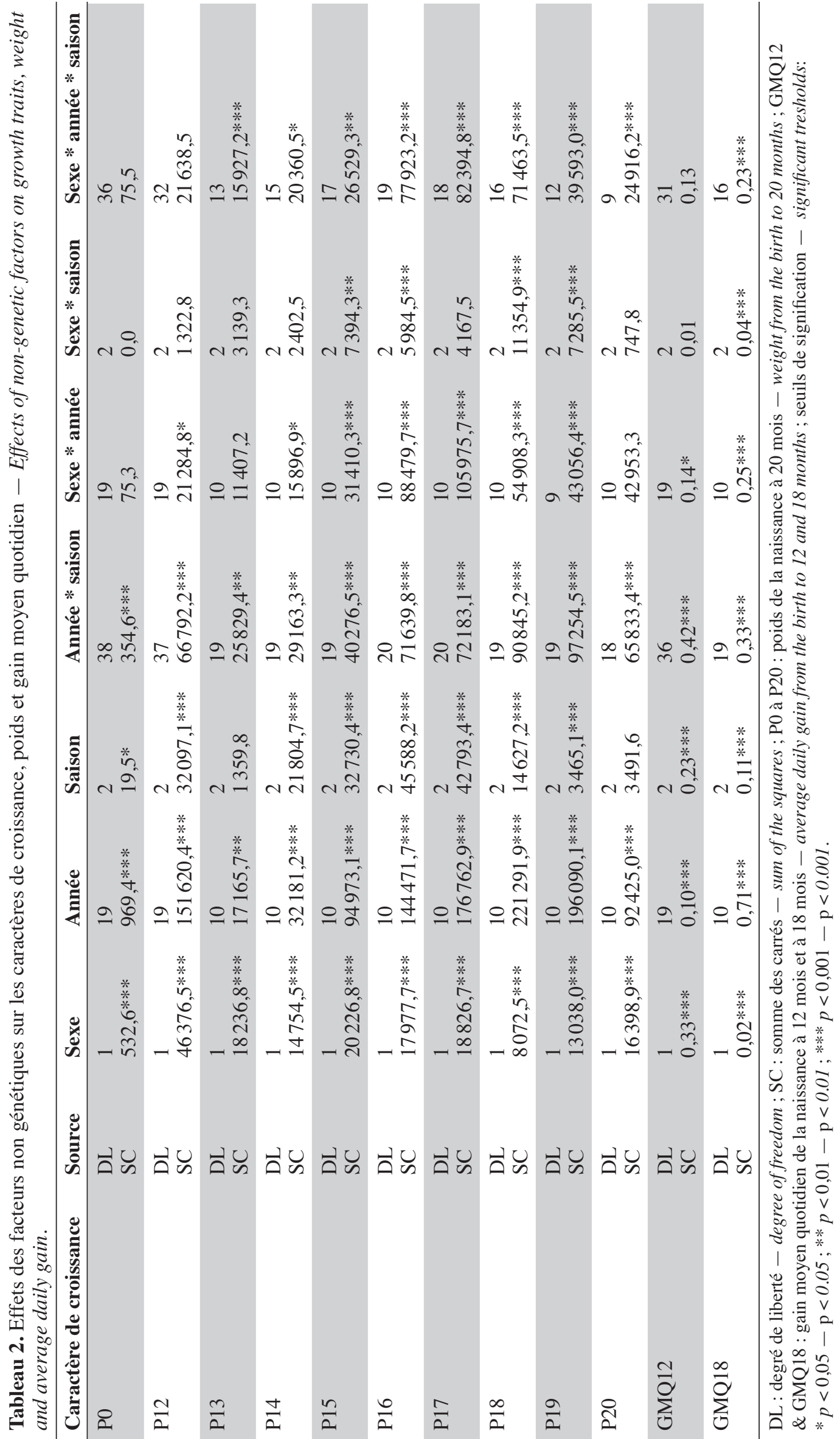




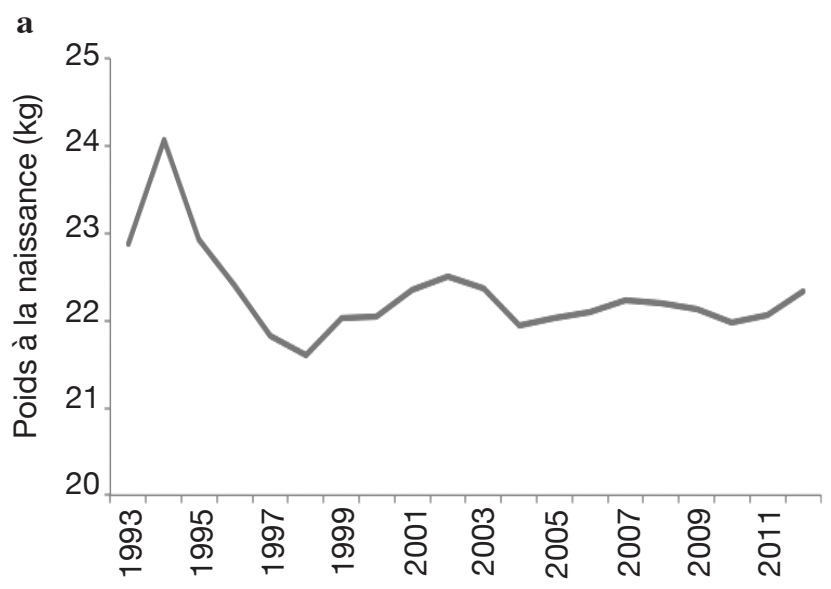

Années de naissance
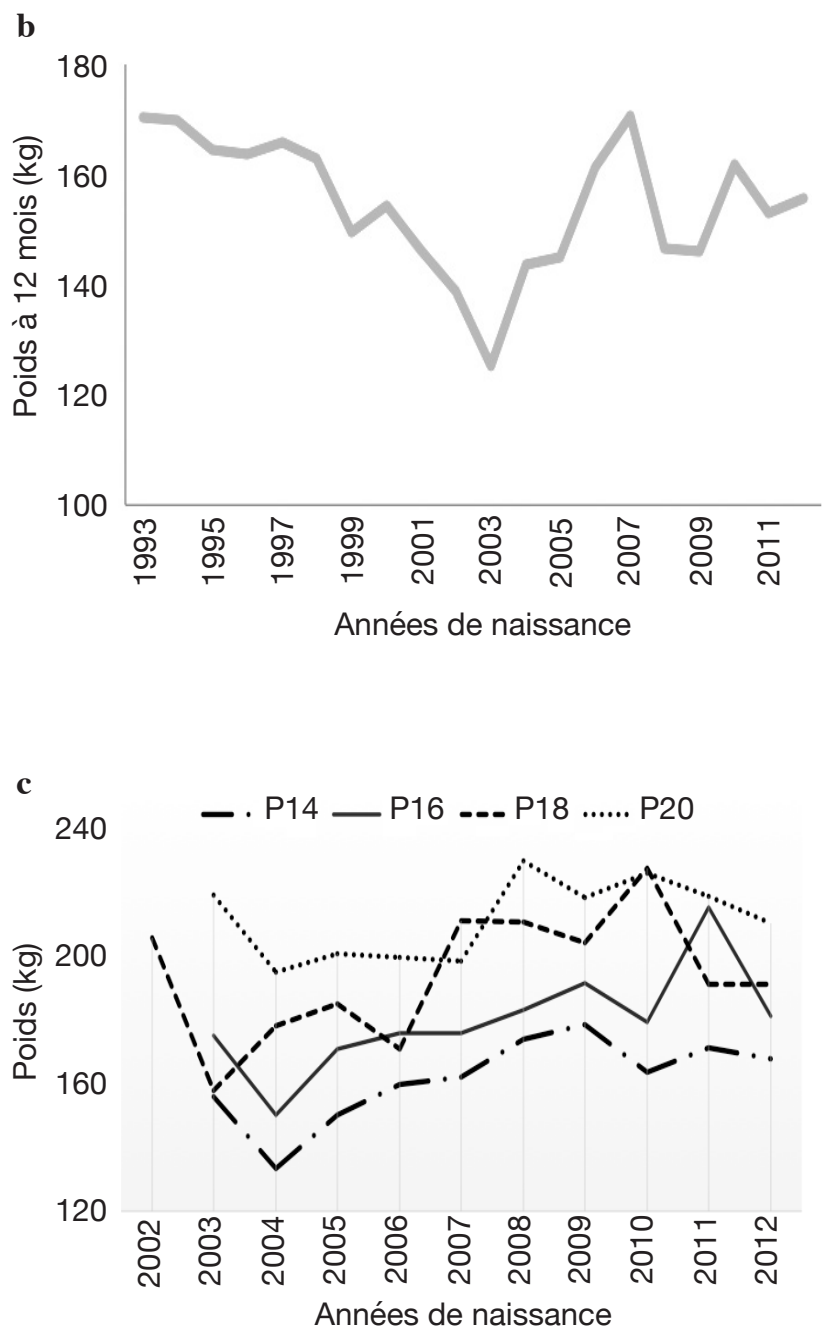

Figure 1. a. Influence de l'année de naissance sur le poids à la naissance - Influence of the birth year on the birth weight; b. Influence de l'année de naissance sur le poids à 12 mois - Influence of the birth year on weight at 12 months ; c. Influence de l'année de naissance sur les poids de 13 à 20 mois (P13 à P20) - Influence of the birth year on the weight between 13 to 20 months (P13 to P20). croissance étudiés. L'interaction entre le sexe et l'année est significative $(p<0,05)$, sauf pour le poids à la naissance, à 13 et 20 mois.

La triple interaction est significative $(p<0,01)$, sauf pour le poids à la naissance et à 12 mois et pour le GMQ12.

\subsection{Paramètres génétiques}

Les valeurs d'héritabilité $\left(\mathrm{h}^{2}\right)$ et des corrélations génétiques (rg) et d'environnement sont données au tableau 3, de même que leurs erreurs standard.

L'héritabilité estimée est modérée pour le poids à la naissance $\left(\mathrm{h}^{2}=0,20\right)$ et la plupart des autres poids $\left(\mathrm{h}^{2}=0,15\right.$ à 0,43$)$ et élevée pour le poids à 13 mois $\left(\mathrm{h}^{2}=0,61\right)$ (Tableau 3). La corrélation génétique est modérée entre les poids à la naissance et à un an (rg = $0,52)$. Les corrélations génétiques entre le poids à la naissance et les poids ultérieurs se réduisent à 0,28 pour le poids à 13 mois et à moins de 0,21 pour les autres. A partir de 12 mois, les corrélations entre les poids consécutifs varient entre 0,67 et 0,93 et les corrélations se réduisent progressivement avec l'espacement des pesées pour atteindre des valeurs inférieures à 0,33 lorsque l'intervalle excède 5 mois (Tableau 3). L'héritabilité des GMQ12 et GMQ18 est élevée, respectivement 0,58 et 0,44 (Tableau 3). La corrélation génétique entre le GMQ12 et le GMQ18 est négative ( $\mathrm{rg}=-0,26)$. Les corrélations entre le GMQ12 et les poids à la naissance, à 12 et 13 mois sont modérées à élevées ( $\mathrm{rg}=0,46$ à 0,66$)$. Les corrélations entre le GMQ18 et les poids sont faibles avant 17 mois $(\mathrm{rg}=0,00$ à 0,26$)$ et modérées à élevées après ( $\mathrm{rg}=$ $0,43$ à 0,77$)$. Les corrélations entre les autres poids et le poids à 17 mois sont particulièrement faibles, à l'inverse ces corrélations avec le poids à 18 mois sont plutôt élevées. Les corrélations environnementales suivent de façon générale les mêmes tendances que les corrélations génétiques. Les héritabilités et les corrélations génétiques et d'environnement sont, à quelques exceptions près, significatives (Tableau 3).

\section{DISCUSSION}

\subsection{Effets des facteurs environnementaux sur les caractères de croissance}

Sexe. Le poids à la naissance et tous les poids de 12 à 20 mois sont significativement supérieurs chez les mâles Azawak $(p<0,001)$. Ces résultats concordent avec ceux obtenus par d'autres auteurs quant à l'effet du sexe sur la croissance des veaux Azawak. Pour le poids à la naissance : Boly et al. (2000) au Burkina Faso, 23,3 $\pm 3,0 \mathrm{~kg}$ pour le mâle et 21,5 $\pm 3,2 \mathrm{~kg}$ chez la femelle; avec ceux de Gouro \& Yenikoye (1991), 
Tableau 3. Héritabilité des caractères de croissance (sur la diagonale), les corrélations génétiques (au-dessus de la diagonale) et les corrélations d'environnement (sous la diagonale) et les erreurs standard (entre parenthèses) - Heritability of growth traits (on the diagonal), genetic correlations (above the diagonal) and environmental correlations (below the diagonal) and standard errors (in brackets).

\begin{tabular}{|c|c|c|c|c|c|c|c|c|c|c|c|c|}
\hline & P0 & P12 & P13 & P14 & P15 & P16 & P17 & P18 & P19 & P20 & GMQ12 & GMQ018 \\
\hline P0 & $\begin{array}{l}\mathbf{0 , 2 0} \\
(\mathbf{0 , 2 0})\end{array}$ & $\begin{array}{l}0,52 \\
(0,03)\end{array}$ & $\begin{array}{l}0,28 \\
(0,03)\end{array}$ & $\begin{array}{l}0,15 \\
(0,03)\end{array}$ & $\begin{array}{l}0,12 \\
(0,03)\end{array}$ & $\begin{array}{l}0,11 \\
(0,02)\end{array}$ & $\begin{array}{l}0,06 \\
(0,03)\end{array}$ & $\begin{array}{l}0,21 \\
(0,02)\end{array}$ & $\begin{array}{l}0,15 \\
(0,02)\end{array}$ & $\begin{array}{c}0,12 \\
(0,02)\end{array}$ & $\begin{array}{c}0,46 \\
(0,01)\end{array}$ & $\begin{array}{c}0,58 \\
(0,02)\end{array}$ \\
\hline P12 & $\begin{array}{l}0,40 \\
(0,01)\end{array}$ & $\begin{array}{l}\mathbf{0 , 1 5} \\
(\mathbf{0 , 0 1})\end{array}$ & $\begin{array}{l}0,91 \\
(0,01)\end{array}$ & $\begin{array}{l}0,49 \\
(0,02)\end{array}$ & $\begin{array}{l}0,44 \\
(0,02)\end{array}$ & $\begin{array}{l}0,38 \\
(0,02)\end{array}$ & $\begin{array}{l}0,00 \\
(0,03)\end{array}$ & $\begin{array}{l}0,53 \\
(0,02)\end{array}$ & & $\begin{array}{c}0,26 \\
(0,02)\end{array}$ & $\begin{array}{c}0,66 \\
(0,01)\end{array}$ & $\begin{array}{c}0,03 \\
(0,02)\end{array}$ \\
\hline P13 & $\begin{array}{l}0,09 \\
(0,04)\end{array}$ & $\begin{array}{l}0,87 \\
(0,02)\end{array}$ & $\begin{array}{l}\mathbf{0 , 6 1} \\
(\mathbf{0 , 0 2})\end{array}$ & $\begin{array}{l}0,78 \\
(0,02)\end{array}$ & $\begin{array}{l}0,68 \\
(0,02)\end{array}$ & $\begin{array}{l}0,53 \\
(0,02)\end{array}$ & $\begin{array}{l}0,10 \\
(0,03)\end{array}$ & $\begin{array}{l}0,64 \\
(0,01)\end{array}$ & $\begin{array}{l}0,34 \\
(0,02)\end{array}$ & $\begin{array}{c}0,34 \\
(0,02)\end{array}$ & $\begin{array}{c}0,55 \\
(0,02)\end{array}$ & $\begin{array}{c}0,07 \\
(0,02)\end{array}$ \\
\hline P14 & $\begin{array}{l}0,14 \\
(0,02)\end{array}$ & $\begin{array}{l}0,37 \\
(0,02)\end{array}$ & $\begin{array}{l}0,69 \\
(0,03)\end{array}$ & $\begin{array}{l}\mathbf{0 , 3 8} \\
(\mathbf{0 , 0 3})\end{array}$ & $\begin{array}{l}0,75 \\
(0,03)\end{array}$ & $\begin{array}{l}0,51 \\
(0,03)\end{array}$ & $\begin{array}{l}0,21 \\
(0,03)\end{array}$ & $\begin{array}{l}0,57 \\
(0,02)\end{array}$ & $\begin{array}{l}0,33 \\
(0,02)\end{array}$ & $\begin{array}{c}0,34 \\
(0,02)\end{array}$ & $\begin{array}{c}0,29 \\
(0,02)\end{array}$ & $\begin{array}{c}0,09 \\
(0,02)\end{array}$ \\
\hline P15 & $\begin{array}{l}0,05 \\
(0,02)\end{array}$ & $\begin{array}{l}0,35 \\
(0,02)\end{array}$ & $\begin{array}{l}0,55 \\
(0,02)\end{array}$ & $\begin{array}{l}0,46 \\
(0,03)\end{array}$ & $\begin{array}{l}\mathbf{0 , 3 8} \\
(\mathbf{0 , 0 2})\end{array}$ & $\begin{array}{l}0,93 \\
(0,01)\end{array}$ & $\begin{array}{l}0,50 \\
(0,02)\end{array}$ & $\begin{array}{l}0,76 \\
(0,02)\end{array}$ & & $\begin{array}{c}0,48 \\
(0,02)\end{array}$ & $\begin{array}{c}0,18 \\
(0,02)\end{array}$ & $\begin{array}{c}0,20 \\
(0,02)\end{array}$ \\
\hline P16 & $\begin{array}{l}0,15 \\
(0,02)\end{array}$ & $\begin{array}{l}0,30 \\
(0,02)\end{array}$ & $\begin{array}{l}0,48 \\
(0,02)\end{array}$ & $\begin{array}{l}0,51 \\
(0,02)\end{array}$ & $\begin{array}{l}0,64 \\
(0,02)\end{array}$ & $\begin{array}{l}\mathbf{0 , 3 6} \\
(\mathbf{0 , 0 1})\end{array}$ & $\begin{array}{l}0,69 \\
(0,03)\end{array}$ & $\begin{array}{l}0,82 \\
(0,02)\end{array}$ & $\begin{array}{l}0,59 \\
(0,02)\end{array}$ & $\begin{array}{c}0,52 \\
(0,02)\end{array}$ & $\begin{array}{c}0,12 \\
(0,02)\end{array}$ & $\begin{array}{c}0,26 \\
(0,02)\end{array}$ \\
\hline P17 & $\begin{array}{l}0,07 \\
(0,03)\end{array}$ & $\begin{array}{l}0,28 \\
(0,03)\end{array}$ & $\begin{array}{l}0,36 \\
(0,03)\end{array}$ & $\begin{array}{l}0,23 \\
(0,03)\end{array}$ & $\begin{array}{l}0,44 \\
(0,03)\end{array}$ & $\begin{array}{l}0,79 \\
(0,02)\end{array}$ & $\begin{array}{l}\mathbf{0 , 4 3} \\
(\mathbf{0 , 0 2})\end{array}$ & $\begin{array}{l}0,80 \\
(0,02)\end{array}$ & $\begin{array}{l}0,62 \\
(0,02)\end{array}$ & $\begin{array}{c}0,52 \\
(0,02)\end{array}$ & & $\begin{array}{c}0,43 \\
(0,02)\end{array}$ \\
\hline P18 & $\begin{array}{l}0,12 \\
(0,01)\end{array}$ & $\begin{array}{l}0,23 \\
(0,01)\end{array}$ & $\begin{array}{l}0,37 \\
(0,02)\end{array}$ & $\begin{array}{l}0,39 \\
(0,02)\end{array}$ & $\begin{array}{l}0,50 \\
(0,02)\end{array}$ & $\begin{array}{l}0,77 \\
(0,01)\end{array}$ & $\begin{array}{l}0,82 \\
(0,01)\end{array}$ & $\begin{array}{l}\mathbf{0 , 3 6} \\
(\mathbf{0 , 0 1})\end{array}$ & $\begin{array}{l}0,74 \\
(0,02)\end{array}$ & $\begin{array}{c}0,64 \\
(0,02)\end{array}$ & $\begin{array}{c}0,22 \\
(0,02)\end{array}$ & $\begin{array}{c}0,46 \\
(0,02)\end{array}$ \\
\hline P19 & $\begin{array}{l}0,10 \\
(0,02)\end{array}$ & $\begin{array}{l}0,17 \\
(0,02)\end{array}$ & $\begin{array}{l}0,26 \\
(0,02)\end{array}$ & $\begin{array}{l}0,26 \\
(0,02)\end{array}$ & $\begin{array}{l}0,39 \\
(0,02)\end{array}$ & $\begin{array}{l}0,58 \\
(0,02)\end{array}$ & $\begin{array}{l}0,61 \\
(0,02)\end{array}$ & $\begin{array}{l}0,90 \\
(0,01)\end{array}$ & $\begin{array}{l}\mathbf{0 , 3 7} \\
(\mathbf{0 , 0 1})\end{array}$ & $\begin{array}{c}0,67 \\
(0,02)\end{array}$ & $\begin{array}{c}0,04 \\
(0,02)\end{array}$ & $\begin{array}{c}0,77 \\
(0,01)\end{array}$ \\
\hline P20 & $\begin{array}{l}0,06 \\
(0,01)\end{array}$ & $\begin{array}{l}0,11 \\
(0,01)\end{array}$ & $\begin{array}{l}0,20 \\
(0,02)\end{array}$ & $\begin{array}{l}0,22 \\
(0,02)\end{array}$ & $\begin{array}{l}0,31 \\
(0,02)\end{array}$ & $\begin{array}{l}0,47 \\
(0,01)\end{array}$ & $\begin{array}{l}0,48 \\
(0,02)\end{array}$ & $\begin{array}{l}0,76 \\
(0,01)\end{array}$ & $\begin{array}{l}0,75 \\
(0,01)\end{array}$ & $\begin{array}{c}\mathbf{0 , 3 7} \\
(\mathbf{0 , 0 1})\end{array}$ & $\begin{array}{c}0,01 \\
(0,02)\end{array}$ & $\begin{array}{c}0,56 \\
(0,02)\end{array}$ \\
\hline GMQ12 & $\begin{array}{l}0,60 \\
(0,01)\end{array}$ & $\begin{array}{l}0,65 \\
(0,01)\end{array}$ & $\begin{array}{l}0,53 \\
(0,02)\end{array}$ & $\begin{array}{l}0,40 \\
(0,02)\end{array}$ & $\begin{array}{l}0,25 \\
(0,02)\end{array}$ & $\begin{array}{l}0,20 \\
(0,02)\end{array}$ & $\begin{array}{l}0,09 \\
(0,03)\end{array}$ & $\begin{array}{l}0,04 \\
(0,02)\end{array}$ & $\begin{array}{l}0,00 \\
(0,02)\end{array}$ & $\begin{array}{l}-0,06 \\
(0,02)\end{array}$ & $\begin{array}{c}\mathbf{0 , 5 8} \\
(\mathbf{0 , 0 1})\end{array}$ & $\begin{array}{l}-0,26 \\
(0,02)\end{array}$ \\
\hline GMQ18 & $\begin{array}{l}0,08 \\
(0,01)\end{array}$ & $\begin{array}{l}0,22 \\
(0,01)\end{array}$ & $\begin{array}{l}0,22 \\
(0,01)\end{array}$ & $\begin{array}{l}0,13 \\
(0,02)\end{array}$ & $\begin{array}{l}0,16 \\
(0,02)\end{array}$ & $\begin{array}{l}0,31 \\
(0,02)\end{array}$ & $\begin{array}{l}0,45 \\
(0,02)\end{array}$ & $\begin{array}{l}0,58 \\
(0,01)\end{array}$ & $\begin{array}{l}0,73 \\
(0,01)\end{array}$ & $\begin{array}{c}0,45 \\
(0,02)\end{array}$ & $\begin{array}{l}-0,05 \\
(0,02)\end{array}$ & $\begin{array}{c}\mathbf{0 , 4 4} \\
(\mathbf{0 , 0 1})\end{array}$ \\
\hline
\end{tabular}

P0 à P20 : poids de la naissance à 20 mois - weight from the birth to 20 months; GMQ12 et GMQ18 : gain moyen quotidien de la naissance à 12 mois et à 18 mois - average daily weight gain from the birth to 12 and 18 months.

au Niger, $28,8 \mathrm{~kg}$ contre $25,5 \mathrm{~kg}$; Naroua et al. (2004), au Niger, 22,0 $\pm 2,1$ et 21,9 $\pm 1,7 \mathrm{~kg}$. Pour le poids à 12 mois : Boly et al. (2000) au Burkina Faso, $132 \pm 24,7 \mathrm{~kg}$ chez le mâle et $126 \pm 26,2 \mathrm{~kg}$ chez la femelle ; Achard \& Chanono (1995) à la station de Toukounous au Niger chez des animaux nés en 1987, 1989 et $1991,253 \pm 5 \mathrm{~kg}$ contre $221 \pm 5 \mathrm{~kg}$ et Boly et al. (2000) au Burkina Faso, $168 \pm 26,05 \mathrm{~kg}$ chez le mâle et $159 \pm 28,13 \mathrm{~kg}$ chez la femelle. Pour les GMQ, Achard \& Chanono (1995) au Niger observent $70 \mathrm{~g} \mathrm{au}$ profit des mâles après l'âge de 6 mois. Toutefois, Boly et al. (2000) n'ont pas observé de différence de GMQ entre 7 et 18 mois.

Saison et année de naissance. La saison et l'année de naissance ont une influence significative sur le poids à la naissance, les poids de 12 à 20 mois et les GMQ à la station de Toukounous. Des effets semblables ont été observés dans les élevages traditionnels par Achard \& Chanono (1995) et Naroua et al. (2004) pour les poids à 9,18 et 24 mois des veaux nés à la station de Toukounous en 1989 et 1991. Ces derniers auteurs ont également observé un ralentissement de la croissance chez les taureaux en saison sèche avec un GMQ de $143 \mathrm{~g}$ et une reprise de la croissance avec des GMQ de l'ordre de $604 \mathrm{~g}$ en saison des pluies où les ressources fourragères sont abondantes. Cependant, cet effet année ou saison, observé dans la présente étude, n'est pas lié à la sècheresse, mais plutôt au déficit de la biomasse fourragère. En effet, les quantités de pluies très supérieures à la moyenne $\left(300 \mathrm{~mm} \cdot \mathrm{cm}^{-1}\right) \mathrm{de}$ 2001 à 2008 ne paraissent pas avoir eu d'impact sur la production fourragère, notamment en 2003 et $2004 \mathrm{au}$ vu des performances de croissance (Figures $\mathbf{1 b}$ et $\mathbf{1 c}$ ). L'effet année est dû à la quantité de biomasse produite qui est plutôt en relation avec une bonne répartition du nombre de jours de pluie.

Contrairement à nos résultats, certains travaux n'ont pas rapporté de différence de poids à la naissance entre la saison des pluies et la saison sèche : Olawumi \& 
Salako (2010) en race White Fulani dans le Sud-Ouest du Nigeria; Mulindwa et al. (2012) à Serere en Uganda en races Sahiwal et Boran. Boly et al. (2000) n'ont pas constaté un effet significatif de la saison de naissance sur le GMQ.

L'influence de l'année et de la saison de naissance et de leur interaction sur la croissance s'explique par leurs effets sur la qualité et la quantité des pâturages qui sont la base pratiquement exclusive de l'alimentation des animaux en croissance et des vaches gestantes à la station de Toukounous. Il est donc important de considérer ces facteurs dans les études des paramètres génétiques des caractères de croissance. Pour atténuer les effets des carences alimentaires sur les paramètres génétiques de croissance, Denis \& Valenza (1971) ont recommandé une supplémentation des veaux à partir de 2 à 3 mois. Selon eux, on ne peut apprécier correctement la croissance des animaux élevés dans les conditions sahéliennes sur parcours naturels sans supplémentation, c'est pourquoi il serait intéressant de reprendre les études sur le bovin Azawak sous une alimentation intensive et à volonté avant le sevrage. Pour d'autres auteurs, l'estimation des paramètres génétiques doit se faire dans des conditions aussi proches que possible des conditions commerciales habituelles ou, dans notre cas, dans des conditions auxquelles les animaux sont adaptés.

\subsection{Paramètres génétiques}

L'héritabilité du poids à la naissance des zébus Azawak estimée dans cette étude est modérée $(0,20 \pm 0,02)$. Néanmoins, cette valeur est inférieure à celles trouvées chez les bovins Azawak à la station de Toukounous $\left(h^{2}=0,30\right)$ par Chartier et al. (1982) et les bovins Brahman au Venezuela $\left(h^{2}=0,33\right)$ (Plasse et al., 2002), chez les bovins Brahman en Afrique du Sud $\left(\mathrm{h}^{2}=0,28\right)$ (Pico et al., 2004), chez les bovins Horo et métis en Éthiopie $\left(\mathrm{h}^{2}=0,68 \pm 0,09\right)$ (Abera et al., 2011) et est supérieure à celle trouvée chez les bovins N'Dama au Nigeria $\left(h^{2}=0,10 \pm 0,05\right)$ (Abdullah \& Olutogun, 2006). Les résultats de la présente étude sont similaires à ceux de Assan (2012) chez les bovins Tuli au Zimbabwe $\left(\mathrm{h}^{2}=0,25 \pm 0,03\right)$ et de Neser et al. (2012) chez les bovins Brangus en Afrique du Sud $\left(h^{2}=0,21 \pm 0,02\right)$. Ces valeurs modérées d'héritabilité pourraient être liées aux effets d'un environnement stressant sur les mères : variation des températures, alimentation médiocre, parasitisme endémique (Goyache \& Gutierrez, 2001). Néanmoins, cette valeur d'héritabilité du poids de naissance indique qu'une sélection sur les performances individuelles pourrait être efficace (Gunawan \& Jakaria, 2011).

L'héritabilité estimée du poids à un an est modérée $\left(h^{2}=0,15 \pm 0,01\right)$. Cette valeur est inférieure à celles rapportées chez le zébu Gobra au Sénégal $\left(h^{2}=\right.$
$0,24 \pm 0,07$ ) (Diop \& Van Wylk, 1998) et chez le bovin Boran en Éthiopie $\left(\mathrm{h}^{2}=0,34 \pm 0,24\right)$ (Haile-Mariam \& Kassa-Mersha, 1995). Cette valeur est supérieure à celle rapportée chez les bovins Brahman en Afrique du Sud $\left(\mathrm{h}^{2}=0,14\right)$ (Pico et al., 2004). Praharani (2009) conclut, de son étude sur le bétail de Bali, que des valeurs modérées à élevées d'héritabilité des poids à la naissance et à un an sont favorables à la réussite d'un programme de sélection sur les performances de croissance pondérale.

Concernant l'héritabilité des poids ultérieurs à un an, nos résultats sont similaires $\left(\mathrm{h}^{2}=0,36\right.$ à 0,61$)$ à ceux de Afroz et al. (2011) qui ont trouvé des valeurs comprises entre 0,44 et 0,50 pour l'héritabilité des poids de 15 à 24 mois. Ils sont supérieurs à ceux de Pico et al. (2004) $\left(\mathrm{h}^{2}=0,18\right)$ entre 16 à 30 mois, de Diop \& Van Wylk (1998) chez le zébu Gobra au Sénégal $\left(h^{2}=\right.$ $0,14 \pm 0,06$ ), de Neser et al. (2012) qui ont obtenu respectivement $h^{2}=0,29$ et $h^{2}=0,24$ pour les poids à 18 mois et supérieur à 18 mois chez le bovin Brangus.

Les héritabilités des GMQ estimées dans cette étude $\left(\mathrm{h}^{2}=0,44\right.$ à 0,58$)$ sont supérieures à celles de Waheed et al. (2003) $\left(\mathrm{h}^{2}=0,01 \pm 0,01\right)$ et de Akpa et al. (2007) $\left(\mathrm{h}^{2}=0,13 \pm 0,15\right)$, mais du même ordre de grandeur que les héritabilités rapportées par Magana \& Segura (1997) entre la naissance et 18 mois $\left(h^{2}=0,56 \pm 0,20\right)$ et par Afroz et al. (2011) de 9 à 24 mois $\left(h^{2}=0,49\right)$.

Les corrélations génétiques entre les différents poids sont modérées à faibles, mais positives, la sélection pour un poids donné entrainera donc une réponse corrélée favorable. Il en est de même pour les corrélations d'environnement, les conditions extensives qui ont prévalu tout au long de notre étude ont eu un effet identique sur tous les poids.

L'héritabilité estimée des poids mesurés de 14 à 20 mois est modérée et homogène et les corrélations environnementales sont positives et fortes, surtout entre poids consécutifs. Une influence des effets environnementaux a été observée par Chartier et al. (1982) sur la croissance pondérale des veaux Azawak à la station de Toukounous, ainsi que dans la présente étude. Pour améliorer la croissance pondérale, il faut donc d'abord agir sur les conditions environnementales à la station de Toukounous.

\section{CONCLUSIONS}

Les facteurs environnementaux dont nous disposons, notamment le sexe, la saison et l'année de naissance, sont une source de variation des caractères de croissance chez le zébu Azawak. Il est donc important de tenir compte de ces facteurs lors de l'estimation des paramètres génétiques et de la prédiction de valeurs d'élevage afin d'éviter les biais. Les estimations de l'héritabilité des caractères de croissance de la 
naissance à 20 mois sont modérées à élevées, ce qui indique qu'un programme de sélection pour la production de viande peut être efficace dans la race Azawak. Étant donné l'importance du poids à la mise à la reproduction et celle-ci étant fixée à deux ans, le poids à 20 mois apparait comme le critère de sélection de choix. D'autant que son héritabilité est de 0,37 , du même ordre que celle des poids mesuré entre 14 et 20 mois, et qu'il présente des corrélations génétiques favorables de plus de 0,50 avec les poids mesurés à partir de 15 mois et avec le GMQ18. Chartier et al. (1982), quant à eux, proposaient le poids à 6 mois comme caractère à sélectionner, car ils avaient trouvé à la station de Toukounous une héritabilité plus élevée pour le poids à 6 mois $(0,70)$, qui présentait en outre des corrélations génétiques et d'environnement élevées avec les autres poids étudiés, aux âges de 12, 24 et 36 mois, mais dont l'héritabilité était de l'ordre de 0,50 .

Les résultats relatifs aux paramètres génétiques et aux effets non génétiques à prendre en compte nous fournissent les moyens d'actualiser un modèle d'évaluation génétique des reproducteurs élevés dans la station de Toukounous. Grâce aux index obtenus, d'une part une sélection de taureaux pour l'insémination artificielle à destination de tous les élevages pourra être opérée et, d'autre part, une stratégie d'accouplements dirigés pourra être mise en place en station. Ainsi, la race Azawak au Niger se verra dotée d'un schéma d'amélioration génétique reposant tant sur la voie mâle que femelle.

\section{Bibliographie}

Abdullah A.R. \& Olutogun O., 2006. Estimates of genetic and phenotypic parameters for preweaning growth traits of N'Dama (Bos taurus) calves in the humid tropics of Nigeria. Livest. Res. Rural Dev., 18, Article \#120.

Abera H., Abegaz S. \& Mekasha Y., 2011. Genetic parameters estimates of pre-weaning weight of Horo (zebu) and their crosses with Holstein Friesian and Jersey cattle breeds in Ethiopia. Int. J. Livest. Prod., 2, 84-91.

Achard F. \& Chanono M., 1995. Un système d'élevage performant bien adapté à l'aridité à Toukounous. Sécheresse, 6, 215-222.

Afroz M.A., Hoque M.A. \& Bhuiyan A.K.F.H., 2011. Estimation of heritability for growth traits of Red Chittagong cattle in a nucleus herd. Bangladesh Veterinarian, 28, 39-46.

Akpa G.N. et al., 2007. Measures of daily weight gain in Friesian-Bunji crossbred heifers and their relationship with first lactation milk yield. Int. J. Dairy Sci., 2, 380386.

Assan N., 2012. Genetic parameters estimation and trends for birth weight in cattle in local cattle breeds of Zimbabwe. J. Anim. Sci. Adv., 2 (suppl. 3.1), 274281.

Boly H. et al., 2000. Reproduction et croissance du zébu Azawak en zone soudano-sahélienne. Ann. Univ. Ouagadougou, 8(Série B), 85-98.

Chartier P., Laoualy A. \& Planchenault D., 1982. Estimation of the different genetic parameters of weight growth in the Azawak zebu. Rev. Elev. Med. Vet. Pays Trop., 35, 413-419.

Denis J.-P. \& Valenza J., 1971. Exteriorization of genetic potentialities of Senegalese Peulh zebu (Gorbra). Rev. Elev. Med. Vet. Pays Trop., 24, 409-418.

Diop M. \& Van Wylk L.D., 1998. Estimates of genetic parameters for growth traits of Gobra cattle. Anim. Sci., 66, 349-355.

Gouro S.A. \& Yenikoye A., 1991. Étude préliminaire sur le comportement d'œstrus et la progestéronémie de la femelle zébu Azawak au Niger. Rev. Elev. Med. Vet. Pays Trop., 44, 100-103.

Goyache F. \& Gutierrez J.P., 2001. Heritability of reproductive traits in Asturiana de los Valles beef cattle breed. Arch. Anim. Breed., 44, 489-496.

Gunawan A. \& Jakaria A., 2011. Genetic and non-genetic effects on birth, weaning and yearling weight of Bali cattle. Media Peternakan, 34(2), 93-98.

Haile-Mariam M. \& Kassa-Mersha H., 1995. Estimates of direct and maternal covariance components of growth traits in Boran cattle. J. Anim. Breed. Genet., 112, 4352.

Kovac M., Groeneveld E. \& Garcia-Cortez A., 2008. Variance components estimation 6.0.2. (VCE-6) User's guide and reference manual. Neustadt, Germany: Institute of Farm Animal Genetics.

Magana J.G. \& Segura J.C., 1997. Heritability and factors affecting growth traits and age at first calving of zebu beef heifers in south-eastern Mexico. Trop. Anim. Health Prod., 29, 185-192.

Mulindwa H.E., Kifaro G.C. \& Sewannyana E., 2012. Comparative pre-weaning growth of zebu cattle and their crosses with Sahiwaland Boran. Uganda J. Agric. Sci., 13, 35-44.

Naroua M., Marichatou H., Vias-Frank G. \& Faye B., 2004. Croissance des veaux Azawak en milieu villageois du Niger. RASPA, 2, 166-169.

Neser F.W.C. et al., 2012. Estimation of genetic parameters for growth traits in Brangus cattle. S. Afr. J. Anim. Sci., 42(suppl.1), 471-473.

Njoya A., Bouchel D., Ngo Tama A.C. \& Planchenault D., 1998. Facteurs affectant le poids à la naissance, la croissance et la viabilité des veaux en milieu paysan au Nord du Cameroun. Rev. Elev. Med. Vet. Pays Trop., 51, 335-343.

Olawumi S.O. \& Salako A.E., 2010. Genetic parameters and factors affecting reproductive performance of white Fulani cattle in South-western Nigeria. Global Veterinaria, 5, 255-258. 
Ouedraogo G.A., Barry M., Toe F. \& Sawadogo G.J., 2008. Évolution de marqueurs biochimiques et endocriniens chez les femelles zébu au cours d'un protocole d'induction de l'œstrus suivi d'une insémination artificielle. Rev. Med. Vet., 159, 169-176.

Pico B.A., Neser F.W.C. \& Van Wylk J.B., 2004. Genetic parameters for growth traits in South African Brahman cattle. S. Afr. J. Anim. Sci., 34(Suppl. 2), 44-46.

Plasse D. et al., 2002. (Co)variance components, genetic parameters and annual trends for calf weights in a Brahman herd kept on floodable savanna. Genet. Mol. Res., 1, 282-297.

Praharani L., 2009. Estimation of direct and maternal effects for weaning and yearling weights in Bali cattle. Indonesian J. Agric., 2, 74-81.
SAS Institute Inc., 2008. SAS Online Doc® 9.1.3. Cary, NC, USA: SAS Institute Inc.

Thiruvenkadan A.K., Panneerselvam S. \& Rajendran R., 2009. Non-genetic and genetic factors influencing growth performance in Murrah Buffalos. S. Afr. J. Anim. Sci., 39(Suppl. 1), 102-106.

Waheed A., Hyder A.U. \& Khan M.S., 2003. Genetic and phenotypic evaluation of growth performance of Bhagnari and Droughtmaster x Bhagnari female calves. Pak. Vet.J., 23, 134-142. 